\title{
Hypoxia in Cell Reprogramming and the Epigenetic Regulations
}

\author{
Nariaki Nakamura ${ }^{1}$, Xiaobing Shi ${ }^{2}$, Radbod Darabi ${ }^{3}$ and Yong $L^{1 *}$ \\ ${ }^{1}$ Department of Orthopaedic Surgery, and Biomedical Engineering, Homer Stryker M.D. School of Medicine, Western \\ Michigan University, Kalamazoo, MI, United States, ${ }^{2}$ Center for Epigenetics, Van Andel Research Institute, Grand Rapids, MI, \\ United States, ${ }^{3}$ The Center for Stem Cell and Regenerative Medicine (CSCRM), Brown Foundation Institute of Molecular \\ Medicine for the Prevention of Human Diseases (IMM), Houston, TX, United States
}

Cellular reprogramming is a fundamental topic in the research of stem cells and molecular biology. It is widely investigated and its understanding is crucial for learning about different aspects of development such as cell proliferation, determination of cell fate and stem cell renewal. Other factors involved during development include hypoxia and epigenetics, which play major roles in the development of tissues and organs. This review will discuss the involvement of hypoxia and epigenetics in the regulation of cellular reprogramming and how interplay between each factor can contribute to different cellular functions as well as tissue regeneration.

Keywords: hypoxia, epigenetic, muscle, cellular reprogramming, stem cells

\section{OPEN ACCESS}

Edited by:

Wei Jiang,

Wuhan University, China

Reviewed by:

Jiayu Chen,

Tongji University, China

Lin Liu,

Nankai University, China

${ }^{*}$ Correspondence:

Yong Li

yong.li@med.wmich.edu

Specialty section:

This article was submitted to

Stem Cell Research,

a section of the journal

Frontiers in Cell and Developmental

Biology

Received: 25 September 2020 Accepted: 06 January 2021 Published: 28 January 2021

Citation:

Nakamura N, Shi X, Darabi R and LiY (2021) Hypoxia in Cell Reprogramming and the Epigenetic Regulations. Front. Cell Dev. Biol. 9:609984. doi: 10.3389/fcell.2021.609984

\section{INTRODUCTION}

When Takahashi and Yamanaka were able to reprogram a fully differentiated skin cell into an induced pluripotent stem cell (iPSC), the promising potential of cellular reprogramming became evident. Through activation of critical transcription factors Oct4, Sox2, Klf4, and c-Myc (Yamanaka factors), any somatic cell could be reprogrammed into a pluripotent stem cell (Takahashi and Yamanaka, 2006; Takahashi et al., 2007). The implications of this discovery are already applied to the development of therapies targeting macular degeneration, spinal cord injuries, acute myeloid leukemia, and more (Chichagova et al., 2018; Maali et al., 2018; Nagoshi et al., 2019). However, the initial method of cellular reprogramming because $<1 \%$ of the cells committed to reprogramming actually gained pluripotency (Takahashi et al., 2007). This inefficiency necessitates that better methods of cellular reprogramming be developed for practical use.

One such method is the culturing of cells in hypoxic conditions. In early development, before the formation of the placenta, the developing embryo is exposed to an environment that varies between 1 and $5 \% \mathrm{O}_{2}$ a concentration much lower than the physiologically normal $20-21 \% \mathrm{O}_{2}$ (Yoshida et al., 2009). When the developing embryo reaches the blastocyst stage, embryonic stem cells (ESCs) are extracted from an inner cell mass (ICM) contained within an outer layer of cells (Thomson et al., 1998; Schrode et al., 2013). ESCs are pluripotent and display high expression of the original Yamanaka factors as well as other transcription factors: Nanog and Lin28A (Moss and Tang, 2003; Pan and Thomson, 2007; Yu et al., 2007).

In an experiment done to test the effect of hypoxia on the production of induced pluripotent stem cells (iPSCs), both mouse embryonic fibroblasts and human dermal fibroblasts were transduced with Yamanaka factors and cultured under normoxia $\left(21 \% \mathrm{O}_{2}\right)$ or hypoxia $\left(5 \% \mathrm{O}_{2}\right)$. Not only did the hypoxia treated cells grow faster, production of iPSCs significantly increased when cells were cultured under $5 \% \mathrm{O}_{2}$ at all periods of induction (Yoshida et al., 2009). In another research, a low oxygen tension $\left(5 \% \mathrm{O}_{2}\right)$ preferentially resulted in the maintenance of a highly 
proliferative, pluripotent population of human ESCs; Moreover, the culture at atmospheric $20 \% \mathrm{O} 2$ levels expressed significantly less OCT4, SOX2, and NANOG than those maintained at 5\% O2 in the hESCs (Forristal et al., 2010).

\section{HYPOXIA AND ITS ROLE IN GENE TRANSCRIPTION}

The initiation of hypoxia results in an upregulation of transcription factors called Hypoxia-Inducible Factors (HIFs). HIFs are activated in response certain environmental conditions, including a low oxygen environment and in inflammation (Lee et al., 2020). HIFs are composed of multiple transcription factors: HIF-1, HIF-2, and HIF-3.

HIF- $1 \alpha$ is the main driver of the cellular response in low oxygen environments. At normal oxygen levels $\left(20-21 \% \mathrm{O}_{2}\right)$, enzymes called prolyl-hydroxylases (PHDs) hydroxylate HIF-1 $\alpha$ at proline residues 402 and 564. This modification tags HIF- $1 \alpha$ for ubiquitination by the E3 ubiquitin ligase complex, which in turn tags it for degradation by the proteasome (Koivunen and Kietzmann, 2018). During hypoxia, the oxygen that is required for hydroxylation, the initial step of degradation, is not present and therefore HIF- $1 \alpha$ is able to remain intact and functional. HIF- $1 \alpha$ accumulates within the cell and dimerizes with the HIF$1 \beta$ subunit forming a heterodimeric HIF- $1 \alpha /$ HIF- $1 \beta$ complex. Afterwards, the complex translocates to the nucleus, where it binds to hypoxia response elements (HREs) and upregulates the expression of its target genes (Sadaghianloo et al., 2020). HIF-1 $\alpha$ has been implicated in the preservation and adjustment of the initial physiological response to hypoxia and plays a vital role in the body's viability in situations where the oxygen level is low. Examples of such adjustments include increasing angiogenesis and glycolysis while decreasing cell proliferation (Majmundar et al., 2010). The HIF-2 mechanism of action works similarly to HIF-1. HIF-2 $\alpha$ is unstable at physiologically normal (20\%) oxygen levels because of hydroxylation at their proline residues. During hypoxia, HIF- $2 \alpha$ dimerizes with HIF- $1 \beta$ and translocates to target genes in the nucleus and upregulates the expression of target genes. The difference between HIF-1 and HIF-2 lies in their target genes (Carroll and Ashcroft, 2006). HIF-2 $\alpha$ binds to HREs upstream of cellular reprogramming genes Oct4, Sox2, Nanog, and significantly increases their expression (Covello et al., 2006; Forristal et al., 2010). HIF-2 $\alpha$ has also been shown to upregulate c-Myc expression by modulating its interactions with cell cycle proteins and is associated with the upregulation of Klf4 (Gordan et al., 2007).

There are many studies indicating that hypoxia regulates gene transcription and expression mostly through the HIF signaling pathways. Hypoxia stimulates the transcription of genes that help to restore oxygen levels or energy productions, in which HIF responds and regulates gene transcription. Hypoxia as an environmental factor can increase muscle stem cells (MuSCs) renewal through its transcription factor HIF-1 $\alpha$ and the Notch pathways (Liu et al., 2012; Yang et al., 2017). There is increasing evidence that hypoxia causes reprogramming of other cells, such as dendritic cells, neural cells, and muscle cells (Bosco and
Varesio, 2012; Mathieu et al., 2013; Vojnits et al., 2015). The HIF1 $\alpha$ pathway is discovered as an enabling regulator of cellular reprogramming through early glycolytic shift and upregulation of PDK1-3 and PKM2. In fact, HIF1 controls the transcription of many target genes to initiate metabolic changes in the early stage and maintains glycolytic metabolism in the later phase of the iPSC reprogramming. Hypoxia is known to enhance the efficiency of reprogramming in the development of iPSCs by upregulating the expression of transcription factors associated with cellular reprogramming and changing the topography of chromatin (Yoshida et al., 2009; Bosco and Varesio, 2012; Mimura et al., 2012; Mathieu et al., 2014; Wang et al., 2016; Alderman et al., 2019).

\section{HYPOXIA AND ITS ROLE IN DEVELOPMENT}

There is a large amount of evidence that hypoxia and HIF play a role in pre-embryonic development (Dunwoodie, 2009). In the developing placenta, the knockdown of HIF- $1 \alpha$ and HIF- $2 \alpha$ results in failure of its formation. Trophoblast invasion, one of essential stages for placentation and pregnancy outcome, likely occurs in a hypoxic environment. Studies have indicated the hypoxia is able to induce 10-11 translocation methylcytosine dioxygenase 1 (TET1) expression that facilitates trophoblast cell migration and invasion through HIF- $1 \alpha$ signaling pathways in the early pregnancy (Koklanaris et al., 2006; Zhu et al., 2017).

More specifically, knockdown of HIF- $1 \alpha$ prevents the fusion of the chorion and the allantois which normally fuse together to form the placenta. Since the chorion and the allantois are not harmed by the knockdown of HIFs, this suggests that HIF plays a role in the integrins that regulate this formation. When exposed to hypoxia, the expression of some of these integrins increased (Dunwoodie, 2009). Additional research suggests that the lack of oxygen and the subsequent upregulation of HIFs plays a vital role in branching morphogenesis, the process that lays down the foundation for the development of key organs such as the nervous system, the respiratory system, the kidney, the salivary glands and the mammary glands (Dunwoodie, 2009; Tsuji et al., 2014). When HIF is knocked down, vascularization of the labyrinthine layer of the placenta decreases, preventing nutrients from the maternal circulatory system to reach the fetal one (Dunwoodie, 2009).

Further along in placental development, HIF is also involved in the formation of the trophoblast (Dunwoodie, 2009). The trophoblast is the outer layer of the developing blastocyst and plays a key role in nourishing the embryo with nutrients. During pre-embryonic development, the trophoblast is attached to the uterine lining and trophoblast cells physically bordering the lining express high amounts of HIF- $1 \alpha$ and HIF- $2 \alpha$. When both subunits are knocked out, the formation of trophoblast subgroups within the trophoblast are inhibited, suggesting that HIF plays a vital role in trophoblast proliferation. As the embryo starts to form into a fetus, hypoxia again plays a major role in the development of major organs. Recent research suggests that HIF also interacts with the Notch signaling pathway. During 
hypoxia, the HIF complex can attach itself to a Notch receptor and induce notch signaling ( $\mathrm{Hu}$ et al., 2014). Notch signaling is implicated in the determination of cell fate and HIF induced notch signaling has proven to be involved in determining blood cell fate as well as determining neural cell fate. Lastly, hypoxia and its corresponding HIFs have shown to play a major role in bone formation, chondrogenesis, heart formation, angiogenesis and formation of the neural crest (Rankin et al., 2011; Lee et al., 2013; Muz et al., 2015; Scully et al., 2016). Overall, HIFs play a significant part in promoting cell proliferation and differentiation of multiple cells during the development of organisms.

\section{HYPOXIA AND ITS ROLE IN DISEASE}

Other than development, hypoxia may play a role in other physiological events characterized by rapid proliferation and differentiation. Many have theorized of a link between the regulation of ESCs and the regulation of cancer stem cells (CSCs). In cancers, the rapid outgrowth of cells and their consumption of oxygen overruns the limited supply of oxygen and creates a hypoxic environment in many areas of the tumor (Muz et al., 2015). The resulting increase in HIFs plays an integral role in cancer pathogenesis by upregulating various transcription factors involved in angiogenesis and cell proliferation (Muz et al., 2015). Some state that this hypoxic environment is also favorable for the cellular reprogramming of non-stem like cancer cells into cancer-stem cells. Heddleston et al. transduced HIF- $2 \alpha$ into nonstem like glioma cells and discovered that the expression of Oct4, Nanog, and c-Myc increased in HIF-2 $\alpha$ transduced cells (Heddleston et al., 2009). The subsequent injection of these cells into immunocompromised mice created significantly larger tumors compared to the control. Further research supports the notion that the chemoresistance of gliomas is also a result of hypoxia induced cellular reprogramming. In tests comparing the sensitivity of glioma associated cells to chemotherapy, adult non-stem like glioma cells showed sensitivity to treatment while glioma stem cells (GSCs) remained unaffected (Wang et al., 2017a). The origin of the GSCs have been debated as some believed that GSCs were derived through contamination from cells already positive for CD133 (GSC marker) while others believed they were a result of hypoxia induced reprogramming of already present $\mathrm{CD}_{133^{-}}$glioma cells. In experiments conducted by Wang et al., they provide evidence it is the latter (Wang et al., 2017b). When CD133- glioma cells were cultured under hypoxia, expression of CD133 increased and signs of GSC behavior such as the ability to asymmetrically divide and the ability to form neurospheres appeared. Hypoxia cultured cells also showed increased expression of ABCG2 and MGMT, proteins associated with increased chemoresistance.

The presence of cells with stem-like properties after hypoxia is not confined to gliomas. Stem-like cells induced from hypoxia have been discovered in cells associated with lung cancer and liver cancer. In non-oncologic fields, increasing amounts of evidence suggest that hypoxia induces the reprogramming of resident muscle cells after injury. Novel multipotent cells have been discovered in the tibialis anterior (TA) muscle in mice after a laceration injury disrupted the vascular structure $(\mathrm{Mu}$ et al., 2011). These cells were termed as injury-induced musclederived stem cells or iMuSCs and have shown the ability to differentiate into cells from all three germ layers and form neurospheres (Vojnits et al., 2015, 2017). Similar cells, termed ischemia-induced multipotent stem cells or iSCs, have also been discovered in the brain of elderly patients after an ischemic stroke (Tatebayashi et al., 2017). The multipotent or pluripotent nature of iMuSCs and iSCs and their presence in tissues after injury point to the therapeutic potential of hypoxia induced cellular reprogramming in muscle and neural regeneration. However, like the aforementioned GSCs, some may argue that the presence of iMuSCs and iSCs can be the result of contamination from circulating cells. In vitro studies that can replicate the formation of such cells through hypoxia or studies identifying the mechanism behind their reprogramming will be useful in clarifying their roles.

\section{Epigenetics in SCNT and iPSCs Reprogramming}

Another major factor involved in the development of stem cells is epigenetics. Epigenetics refers to the cellular machinery that controls gene expression and occurs through the addition or deletion of epigenetic modifications (Handy et al., 2011). Epigenetic modifications refer to any heritable modifications that are made to the DNA or histones. The most basic of these are DNA and histone methylation, but other forms of modification can occur in histones such as acetylation, phosphorylation, ubiquitination, sumoylation etc. These modifications alter the accessibility of chromatin to transcription factors and polymerases that play a role in the transcription of DNA and expression of genes (Handy et al., 2011). Epigenetic modifications play major roles in development and in the development of disease (Portela and Esteller, 2010).

DNA methylation in general exists as a suppression mechanism for many genes. As organisms develop from the embryo into a fetus, areas in the genome with large amounts of CG dinucleotide repeats, known as $\mathrm{CpG}$ islands, experience methylation on the cytosine residue (Portela and Esteller, 2010; Handy et al., 2011). This hypothetically serves to suppress pluripotency at critical genes as pluripotent embryonic stem cells differentiate into their preferred cells and organ systems. The methyl groups block transcription by blocking the attachment of transcription factors onto the DNA segment. Interestingly, methyl groups have also shown to block HIF-1 from attaching to its HRE to regulate erythropoietin transcription (Handy et al., 2011). In addition to blocking transcription factors and polymerases, methyl groups are targeted by MeCP2 proteins, which recruit histone deacetylases (HDACs) to condense the chromatin and prevent transcription (Portela and Esteller, 2010; Handy et al., 2011).

Histones modifications have wide-ranging effects. Histones serve as molecular chaperones and organize DNA into structures called nucleosomes, which consist of 5 subunits, H1, H2A, H2B, $\mathrm{H} 3$, and H4. The subunits $\mathrm{H} 2 \mathrm{~A}, \mathrm{H} 2 \mathrm{~B}, \mathrm{H} 3$, and $\mathrm{H} 4$ consist of the core proteins and are bound together to form "beads" in 
which DNA strands wrap around. $\mathrm{H} 1$ serves as the support that keeps the DNA strands and the core histones in place. The number of histone subunits relates directly to the diversity of epigenetic modifications found in histones. Each core histone subunit has a multitude of modifications the most common of which are found in H3 (Handy et al., 2011). H3 modifications are widely studied and have a significant impact on the activation and repression of genes. The nomenclature of these modifications proceeds in the following order: the subunit of the histone, the amino acid affected, the position of the amino acid, and the type of modification applied. For instance, H3K27me3 indicates a trimethylation at $\mathrm{H} 3$ on Lysine 27 . Some commonly observed modifications on $\mathrm{H} 3$ are $\mathrm{H} 3 \mathrm{~K} 4 \mathrm{me} 1, \mathrm{H} 4 \mathrm{~K} 4 \mathrm{me} 3, \mathrm{H} 3 \mathrm{~K} 36 \mathrm{me} 3$, H3K79me2, H3K9Ac, H3K27Ac, all of which are associated with the opening up of the chromatin structure and gene activation by transcription factors and polymerase. In contract, $\mathrm{H} 3 \mathrm{~K} 9 \mathrm{me} 3$ and $\mathrm{H} 3 \mathrm{~K} 27 \mathrm{me} 3$ are associated with the condensation of chromatin and the blocking of gene expression. It has been found that $\mathrm{H} 3 \mathrm{~K} 27 \mathrm{me} 3$ imprinting defects impede post-implantation development (Matoba et al., 2018). H3K9me3 and H3K4me3 might also affect transcriptional reprogramming and thus impair the developmental potential of SCNT embryos (Matoba and Zhang, 2018). H3K9me3 also showed to be implicated in the majority of barriers to the Somatic Cell Nuclear Transfer (SCNT) and iPSCs reprogramming. Those data suggest the essential, diverse set of roles of epigenetics in cellular reprogramming (Wang et al., 2018).

\section{Epigenetic Modifications in Stem Cell Potency}

Histone modifications are involved in development to set up genes for activation during lineage commitment by H3K4me3 and to repress lineage control genes to maintain pluripotency by H3K27me3 (Shipony et al., 2014). The balance and interaction between these pathways are essential for stem cell homeostasis and are directly linked to cellular behaviors. H3K27me3 and PRC2 each contribute to epigenetically transmitting the memory of repression across generations and during development (Juan et al., 2011; Stojic et al., 2011). H3K27me3 and H3K4me3 promoter bivalency are observed in stem cells and their differentiation, which include embryonic and iPSCs (Liu et al., 2013, 2016; Leschik et al., 2015). Bivalency has a prominent role in post-implantation embryonic development or post-natal organ growth. However, the role of epigenetic regulation in the function of adult tissue stem cells is less well-understood. It has been discovered $\mathrm{H} 3 \mathrm{~K} 27 \mathrm{ac}$ (acetylation) regulates cell reprogramming and mouse ES cell differentiation as well as MyoD expression (Mattout et al., 2011; Khilji et al., 2018; Martire et al., 2019). The effects of epigenetic dysregulation on adult stem cell function depend on the tissue type and the epigenetic regulator affected. Recently studies have increased our understanding of the epigenetic regulations during MuSCs maintenance, activation, differentiation, and homeostasis (Figure 1) (Liu et al., 2013; Kosan et al., 2018).

In the studies of bone marrow mesenchymal stem cells (MSCs), results indicate that hypoxia could induce senescence of MSCs via altered gut microbiota (Xing et al., 2018). However, most reports suggested that hypoxia inhibits senescence of MSCs and maintains their properties (Tsai et al., 2011; Kwon et al., 2017; Korski et al., 2019). In the cardiovascular system, hypoxia involves resident cell senescence either via promotion or prevention of the processes, suggesting the signaling pathways are complex (Korski et al., 2019; Lewinska et al., 2020). It also suggests that the level of $\mathrm{O} 2$ may determine the final role of hypoxic environment. Epigenetic events are essential to establish and maintain the distinct cell lineages and have been shown to be crucial players controlling the fate and function of MSCs and MuSCs(Nakade et al., 2017; Zhang et al., 2019). More recently, a study showed MuSCs forced to express Msx1 exhibited increased proliferation index as well as promoted SSEA1 and Pax7 expression, but restricted MyoD expression and accessed osteogenic genes (Ding et al., 2017).

\section{HYPOXIA IMPACTS HISTONE MODIFICATION AND EPIGENETIC REGULATION}

The Polycomb repressive complex 2 or PRC2 is a histone methyltransferase complex and functions as a transcriptional corepressor (Lee et al., 2006). PRC2 consists of three subunits, SUZ12, EED, and EZH2. EZH2 is the catalytic subunit of PRC2 that can mono-, di-, and trimethylate H3K27 (Conway et al., 2015). In ESCs, PRC2 has been shown to occupy genes that are essential for differentiation and development, and represses those genes by depositing the repressive H3K27me3 mark. When cells undergo differentiation, these genes exhibit a loss of PRC2 occupancy and a loss H3K27 trimethylation. Depletion of PRC2 or its associated subunits in ESCs or mice results in the disappearance of pluripotency, the displacement of $\mathrm{H} 3 \mathrm{~K} 27 \mathrm{me} 3$, and premature differentiation during early development (Koppens et al., 2016; Shan et al., 2017). PRC2 KO mice are non-viable and die early in development (Pasini et al., 2004). These results suggest that PRC2 plays a crucial role during early development by repressing select developmental genes and maintaining ESC pluripotency.

PRC2 subunits are frequently mutated in cancers (Lee et al., 2006; Conway et al., 2015; Laugesen et al., 2016; Veneti et al., 2017). These mutations are observed in a variety of cancers such as breast cancer, colorectal cancer, hepatocellular carcinoma and glioblastoma. Most of the PRC2 mutations are inactivation mutations, leading to downregulation of $\mathrm{H} 3 \mathrm{~K} 27 \mathrm{me} 3$ levels. While H3K27me3 is downregulated, it is never fully eliminated and in fact may appear in greater concentrations in select areas of the genome. In some other cancers, such as lymphomas, EZH2 has hot-spot gain of function mutations. In this case, levels of $\mathrm{H} 3 \mathrm{~K} 27 \mathrm{me} 3$ actually increased and differentiation is blocked. This points to the idea that oncogenic PRC2 mutations redistribute $\mathrm{H} 3 \mathrm{~K} 27 \mathrm{me} 3$ from genes that cause cancer and redistribute them toward genes that control differentiation (Figure 2) (Conway et al., 2015).

Another histone methylation notable for its involvement in pluripotency is $\mathrm{H} 3 \mathrm{~K} 4 \mathrm{me} 3$. $\mathrm{H} 3 \mathrm{~K} 4 \mathrm{me} 3$ is an active mark 


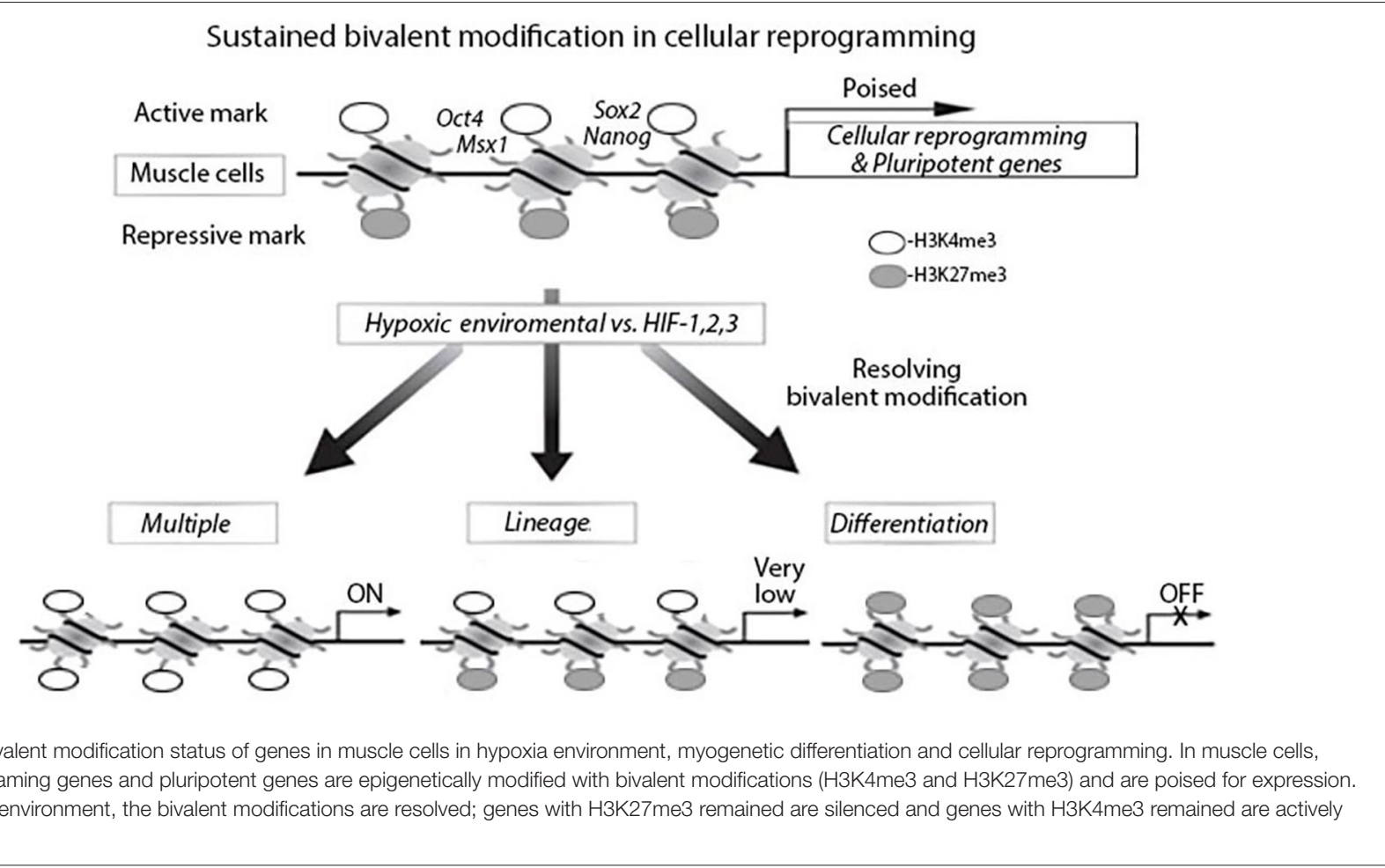

enriched at gene promoters. There is a set of genes that contain both H3K4me3 and H3K27me3 on their promoters, known as bivalent domains (Bernstein et al., 2006). The presence of both H3K4me3 and H3K37me3 at the developmental regulator genes puts these genes under bivalent epigenetic control. When ESCs undergo differentiation, H3K27me3 disappear from the region and is increasingly concentrated with the activating $\mathrm{H} 3 \mathrm{~K} 4 \mathrm{me}$. On a fully differentiated gene, its promoter is only populated with H3K4me3. Downregulation of H3K27me3 via EZH2 knockout in ESCs or muscle stem cells forces the cell to undergo differentiation (Yu et al., 2018; Wang et al., 2019). These findings suggest that bivalent chromatin plays a critical role in the maintenance of stem cell pluripotency and regulation of stem cell differentiation. There are multiple interactions of epigenetic regulations during cellular reprogramming of muscle stem cells in a hypoxia environment. Hypoxia vs. active HIF$1 \alpha$ can increase genome-wide bivalent epigenetic making that could be an initial stimulator in cellular reprogramming. These epigenetic modifications and chromatin states may not only regulate cellular reprogramming but also normalize the multiple differentiation in a different way to support muscle regeneration (Liu et al., 2013; Faralli et al., 2016).

\section{Hypoxia Influences Epigenetics Modifications in Cellular Reprogramming}

Recent studies have shown that hypoxia can affect the level of epigenetic modifications in cells. In breast epithelial adenocarcinoma MCF7 cells, hypoxia increased the levels of H3K27me3 globally and increased the number of genes under bivalent epigenetic control by increasing levels of
H3K4me3 in H3K27me3 concentrated areas (Prickaerts et al., 2016). When reoxygenated, many of the genes retained the bivalency and conferred an epigenetic profile similar to those of embryonic stem cells. Activity of the H3K27me3 demethylase KDM6B/JMJD3 decreased as well, indicating hypoxic control of H3K27me3 demethylation (Prickaerts et al., 2016). In HeLa cells and human fibroblasts, hypoxia increases the levels of H3K4me3 in enhancers of genes associated with cell division and oxidative phosphorylation (Batie et al., 2019). The increase in histone marks at select genes associated with cell proliferation and differentiation matches favorably with the increase in transcription factors, Oct4, Sox2, and Nanog associated with cellular reprogramming. In fact, the addition of Oct4, Sox2, and Nanog to mesenchymal stem cells results in an increase in cell proliferation (Han et al., 2014; Park et al., 2019). A similar increase in cell proliferation was seen when treating mesenchymal stem cells under hypoxia (Kwon et al., 2017).

The co-occupancy of transcription factors suggest the existence of a relationship between hypoxia and epigenetics when regulating cellular reprogramming (Figure 3). Several studies have studied this relationship. One example is the role of hypoxia in Epithelial-mesenchymal transition (EMT). EMT is the process that occurs when epithelial cells undergo reprogramming to become mesenchymal stem cells (Larue and Bellacosa, 2005). Mesenchymal stem cells are multipotent and play crucial roles in early embryonic development and the development of organs. Under hypoxia, promoters of epithelial genes e-cadherin and plakoglobin display decreased levels of $\mathrm{H} 3 \mathrm{~K} 4$ acetylation (H3K4ac) and increased levels of H3K27me3 (Wu et al., 2011). In contrast, promoters of mesenchymal genes 


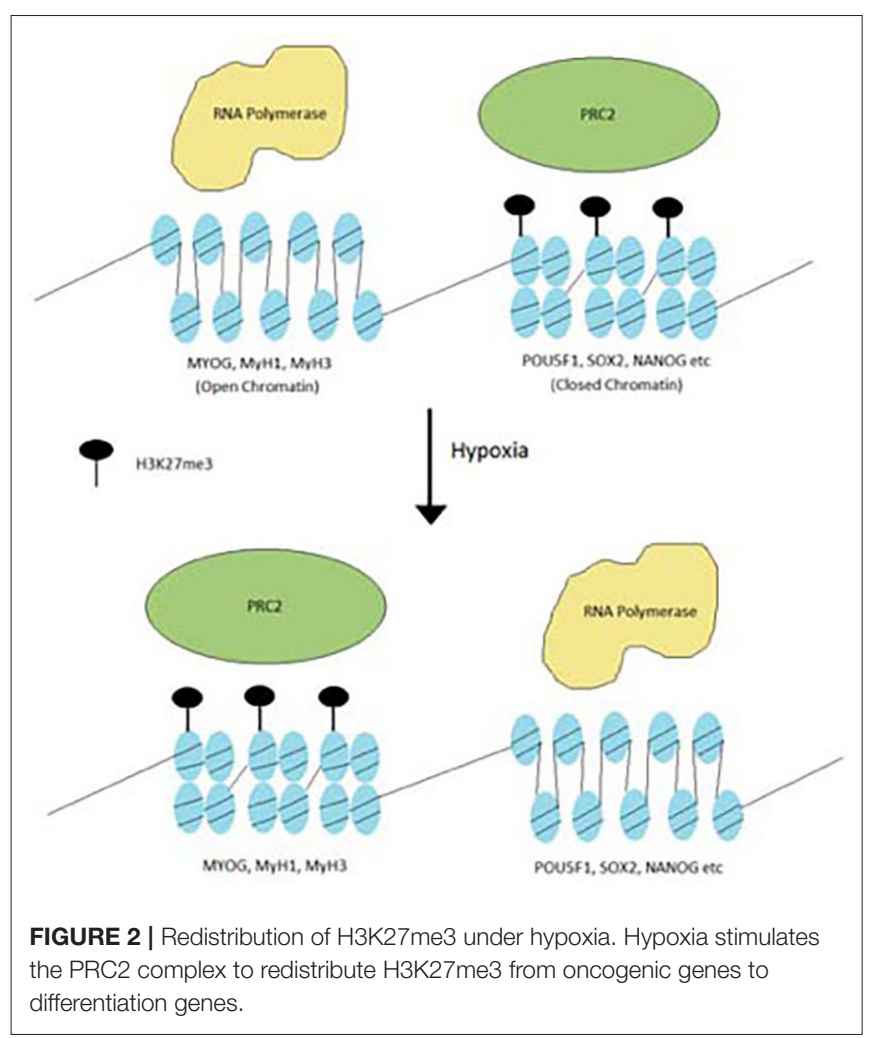

$\mathrm{N}$-cadherin and vimentin showed decreased levels of H3K27me3. In the reprogramming of cancer stem cells such as GSCs, hypoxia has shown to induce the activity of TET1 and TET3 (Prasad et al., 2017). TET1 and TET3 bind to Oct 4 and Nanog regulatory regions and increase the activity of each transcription factor, thereby promoting the reprogramming of glioma cells to glioma stem cells.

Another promising area of research is the impact of hypoxia on the regulation of telomerase and the telomere. Telomeres are linear guanine-rich DNA structures at the ends of chromosomes, and their length determines the behaviors and life in many cells such as germ cells, cancer cells, and pluripotent stem cells. Telomeric DNA is synthesized by way of the ribonucleoprotein called telomerase, which contains a reverse transcriptase (TERT) subunit and an RNA component. Telomeres and TERT are essentially in iPSCs induction and maintenance (Teichroeb et al., 2016). TERT is highly conserved across species and ubiquitously present in pluripotent cells. Moreover, the TERT transcription can be enhanced by DNA methylation at the TERT-DMR via binding to nuclear lamina during cellular reprogramming (Takasawa et al., 2018). Hypoxia can promote telomerase TERT expression and HIF- $1 \alpha$ mediates upregulation of TERT (Yu et al., 2006; Song et al., 2020). This has a significant impact on cellular reprogramming from somatic cells into pluripotent stem cells.

\section{Other Epigenetic Factors in Hypoxia}

While the great majority of this review is focused on histone methylation, the potential role of histone acetylation and cellular reprogramming should not be ignored. An example of the

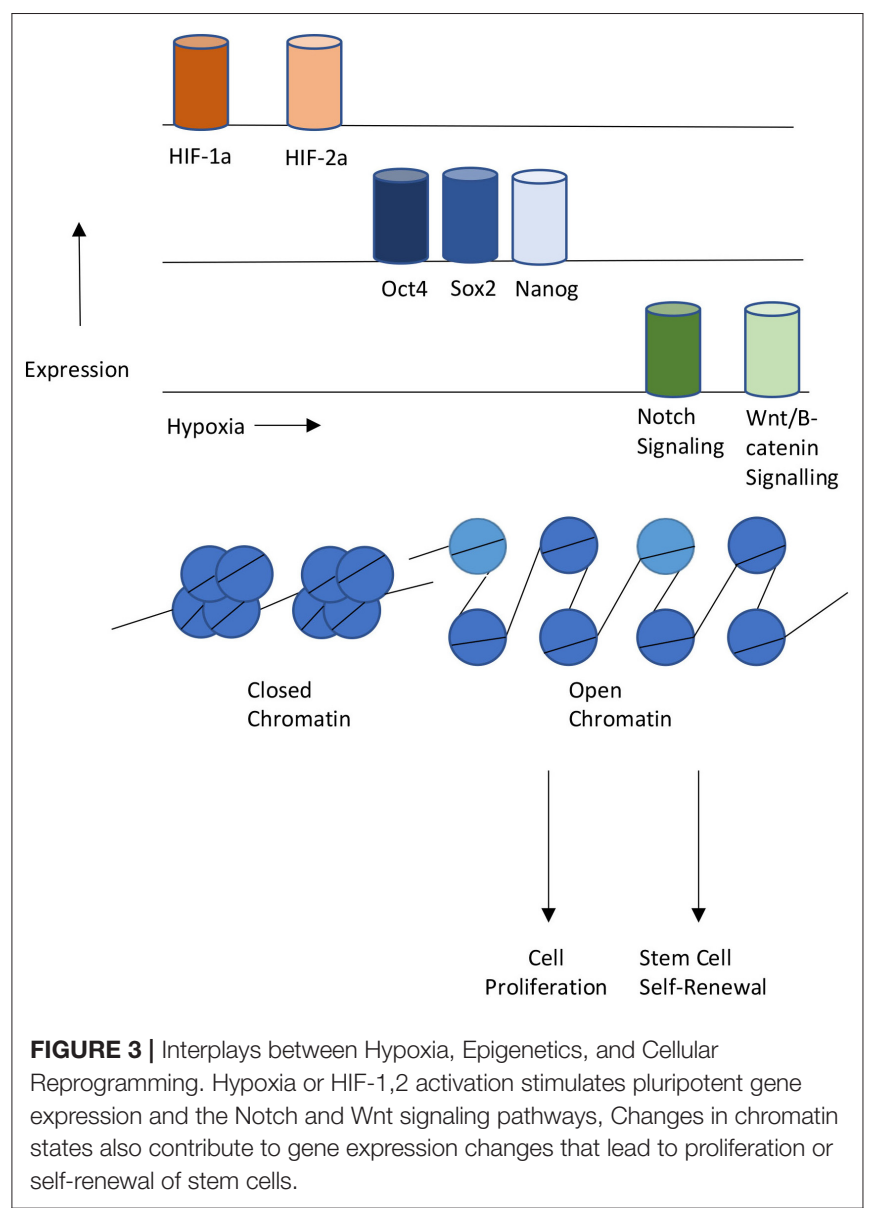

impact of acetylation is the rapid acetylation that occurs after fertilization with the somatic nucleus. Before conception, the histones of mouse oocytes do not display any acetylation (Kim et al., 2003). However, if the same oocyte is implanted with a nucleus from a somatic cell, the histones become rapidly acetylated. This procedure is known as SCNT and through this process a permanently differentiated somatic cell undergoes cellular reprogramming to become a totipotent stem cell (Wilmut et al., 1997, 2002). This rapid acetylation that occurs after fertilization with the somatic nucleus indicates that acetylation plays a role in cellular reprogramming. More recently, a report suggests that reprogramming of $\mathrm{H} 3 \mathrm{~K} 9 \mathrm{ac}$ is important for optimal SCNT efficiency and identifies Dux as a crucial transcription factor in this process (Yang et al., 2020). Despite the rapid acetylation, many of the fertilized oocytes reverted back to their previous unacetylated state $1-3 \mathrm{~h}$ after fertilization and the reprogramming is inefficient. In a study done by Rybouchkin et al. only 34\% of the oocytes reached the blastocyst stage. However, when a histone decacetylase inhibitor (Trichostatin) was introduced during fertilization, a staggering $81 \%$ of the treated oocytes were able to reach the blastocyst stage compared to $41 \%$ of the non-treated oocytes (Rybouchkin et al., 2006). Another histone deacetylase inhibitor, valproic acid, found similar results (Huangfu et al., 2008). These studies 
indicate that inhibition of histone deacetylation can enhance cellular reprogramming.

Hypoxia has been proven to modulate histone acetylation by increasing histone acetylation at $\mathrm{H} 3$ and regulating the downstream processes of many genes. In addition, the initiation of hypoxia and induction of HIF- $1 \alpha$ can increase the acetylation of $\mathrm{H} 3$ and $\mathrm{H} 4$ in neuroblastoma cells and increases the aggressiveness of neuroblastomas (Poljakova et al., 2014). A potential, exciting future area of research would be investigation to see if histone acetylation can play a role in cellular reprogramming of cancer cells to cancer stem cells.

\section{LOOKING AHEAD}

Hypoxia stimulation greatly affects gene expression and is considered to play an essential role in early embryo development, cell differentiation, and cellular reprogramming. Meanwhile, new techniques have been introduced to this field, such as the development of next-generation sequencing, single cell RNA sequencing, and micro-omics technologies. Recent studies have clearly depicted certain epigenetic changes, including DNA methylation, hydroxymethylation, histone modifications, organoids and 3D structure formation during early embryo development, iPSCs, and somatic cell reprogramming. There is potential for the use of the interplay of hypoxia and epigenetics in regenerative medicine. The existence of reprogrammed cells after hypoxia has been reported in multiple different types of tissue and organs (Shyh-Chang et al., 2013; Mosteiro et al., 2016). These conditional ischemic events via low oxygen stimulation are an intriguing prospect for future investigation. They suggest

\section{REFERENCES}

Alderman, S. L., Crossley, D. A. II, Elsey, R. M., and Gillis, T. E. (2019). Hypoxiainduced reprogramming of the cardiac phenotype in American alligators (Alligator mississippiensis) revealed by quantitative proteomics. Sci. Rep. 9:8592. doi: 10.1038/s41598-019-45023-3

Batie, M., Frost, J., Frost, M., Wilson, J. W., Schofield, P., and Rocha, S. (2019). Hypoxia induces rapid changes to histone methylation and reprograms chromatin. Science 363, 1222-1226. doi: 10.1126/science.aau5870

Bernstein, B. E., Mikkelsen, T. S., Xie, X., Kamal, M., Huebert, D. J., Cuff, J., et al. (2006). A bivalent chromatin structure marks key developmental genes in embryonic stem cells. Cell 125, 315-326. doi: 10.1016/j.cell.2006. 02.041

Bosco, M. C., and Varesio, L. (2012). Dendritic cell reprogramming by the hypoxic environment. Immunobiology 217, 1241-1249. doi: 10.1016/j.imbio.2012.07.023

Carroll, V. A., and Ashcroft, M. (2006). Role of hypoxia-inducible factor (HIF)lalpha versus HIF-2alpha in the regulation of HIF target genes in response to hypoxia, insulin-like growth factor-I, or loss of von Hippel-Lindau function: implications for targeting the HIF pathway. Cancer Res. 66, 6264-6270. doi: 10.1158/0008-5472.CAN-05-2519

Chichagova, V., Hallam, D., Collin, J., Zerti, D., Dorgau, B., Felemban, M., et al. (2018). Cellular regeneration strategies for macular degeneration: past, present and future. Eye 32, 946-971. doi: 10.1038/s41433-018-0061-z

Conway, E., Healy, E., and Bracken, A. P. (2015). PRC2 mediated H3K27 methylations in cellular identity and cancer. Curr. Opin. Cell Biol. 37, 42-48. doi: 10.1016/j.ceb.2015.10.003

Covello, K. L., Kehler, J., Yu, H., Gordan, J. D., Arsham, A. M., Hu, C. J., et al. (2006). HIF-2alpha regulates Oct-4: effects of hypoxia on stem cell that a low oxygen environment may contribute or regulate the processes of tissue regeneration (Fang et al., 2018; Huels and Medema, 2018; Yui et al., 2018). There is undeniable involvement of HIF signaling and epigenetics during events that determine cell fate and cause rapid proliferation and differentiation. There is also evidence of epigenetic modifications at play in muscle regeneration and during differentiation of primary myogenic stem cells (Liu et al., 2013; Faralli et al., 2016). These factors and their association with tissue regeneration is potentially a new field for further study. Additionally, epigenetics have played a significant role in recent drug design (Harrison, 2012). Obviously, there will be a large benefit from the use of these epigenetic drugs or peptides to directly modulate the epigenome and normalize tissue regeneration.

\section{AUTHOR CONTRIBUTIONS}

NN drafted the manuscript and created the figures. XS discussed and edited the manuscript. RD revised and edited the manuscript. YL searched literature, structured, modified figures, and wrote and edited the manuscript. All author approved the final version of the manuscript.

\section{ACKNOWLEDGMENTS}

The authors thank Drs. Juliana Overbey for their critical discussion and help to edit this manuscript. The review is partially supported from the Homer Stryker MD School of Medicine. function, embryonic development, and tumor growth. Genes Dev. 20, 557-570. doi: 10.1101/gad.1399906

Ding, K., Liu, W. Y., Zeng, Q., Hou, F., Xu, J. Z., and Yang, Z. (2017). Msx1-modulated muscle satellite cells retain a primitive state and exhibit an enhanced capacity for osteogenic differentiation. Exp. Cell Res. 352, 84-94. doi: 10.1016/j.yexcr.2017.01.016

Dunwoodie, S. L. (2009). The role of hypoxia in development of the Mammalian embryo. Dev. Cell 17, 755-773. doi: 10.1016/j.devcel.2009.11.008

Fang, L., El Wazan, L., Tan, C., Nguyen, T., Hung, S. S. C., Hewitt, A. W., et al. (2018). Potentials of cellular reprogramming as a novel strategy for neuroregeneration. Front. Cell. Neurosci. 12:460. doi: 10.3389/fncel.2018. 00460

Faralli, H., Wang, C., Nakka, K., Benyoucef, A., Sebastian, S., Zhuang, L., et al. (2016). UTX demethylase activity is required for satellite cell-mediated muscle regeneration. J. Clin. Invest. 126, 1555-1565. doi: 10.1172/JCI83239

Forristal, C. E., Wright, K. L., Hanley, N. A., Oreffo, R. O., and Houghton, F. D. (2010). Hypoxia inducible factors regulate pluripotency and proliferation in human embryonic stem cells cultured at reduced oxygen tensions. Reproduction 139, 85-97. doi: 10.1530/REP-09-0300

Gordan, J. D., Bertout, J. A., Hu, C. J., Diehl, J. A., and Simon, M. C. (2007). HIF2alpha promotes hypoxic cell proliferation by enhancing c-myc transcriptional activity. Cancer Cell 11, 335-347. doi: 10.1016/j.ccr.2007.02.006

Han, S. M., Han, S. H., Coh, Y. R., Jang, G., Chan Ra, J., Kang, S. K., et al. (2014). Enhanced proliferation and differentiation of Oct4- and Sox2-overexpressing human adipose tissue mesenchymal stem cells. Exp. Mol. Med. 46:e101. doi: $10.1038 / \mathrm{emm} .2014 .28$

Handy, D. E., Castro, R., and Loscalzo, J. (2011). Epigenetic modifications: basic mechanisms and role in cardiovascular disease. Circulation 123, 2145-2156. doi: 10.1161/CIRCULATIONAHA.110.956839 
Harrison, C. (2012). Structure-based drug design: opening the door to an epigenetic target. Nat. Rev. Drug Discov. 11:672. doi: 10.1038/nrd3827

Heddleston, J. M., Li, Z., McLendon, R. E., Hjelmeland, A. B., and Rich, J. N. (2009). The hypoxic microenvironment maintains glioblastoma stem cells and promotes reprogramming towards a cancer stem cell phenotype. Cell Cycle 8, 3274-3284. doi: 10.4161/cc.8.20.9701

Hu, Y. Y., Fu, L. A., Li, S. Z., Chen, Y., Li, J. C., Han, J., et al. (2014). Hif-1alpha and Hif-2alpha differentially regulate Notch signaling through competitive interaction with the intracellular domain of Notch receptors in glioma stem cells. Cancer Lett. 349, 67-76. doi: 10.1016/j.canlet.2014.03.035

Huangfu, D., Osafune, K., Maehr, R., Guo, W., Eijkelenboom, A., Chen, S., et al. (2008). Induction of pluripotent stem cells from primary human fibroblasts with only Oct4 and Sox2. Nat. Biotechnol. 26, 1269-1275. doi: 10.1038/nbt.1502

Huels, D. J., and Medema, J. P. (2018). Think about the environment: cellular reprogramming by the extracellular matrix. Cell Stem Cell 22, 7-9. doi: 10.1016/j.stem.2017.12.006

Juan, A. H., Derfoul, A., Feng, X., Ryall, J. G., Dell'Orso, S., Pasut, A., et al. (2011). Polycomb EZH2 controls self-renewal and safeguards the transcriptional identity of skeletal muscle stem cells. Genes Dev. 25, 789-794. doi: 10.1101/gad.2027911

Khilji, S., Hamed, M., Chen, J., and Li, Q. (2018). Loci-specific histone acetylation profiles associated with transcriptional coactivator p300 during early myoblast differentiation. Epigenetics 13, 642-654. doi: 10.1080/15592294.2018.1489659

Kim, J. M., Liu, H., Tazaki, M., Nagata, M., and Aoki, F. (2003). Changes in histone acetylation during mouse oocyte meiosis. J. Cell Biol. 162, 37-46. doi: $10.1083 /$ jcb.200303047

Koivunen, P., and Kietzmann, T. (2018). Hypoxia-inducible factor prolyl 4-hydroxylases and metabolism. Trends Mol. Med. 24, 1021-1035. doi: 10.1016/j.molmed.2018.10.004

Koklanaris, N., Nwachukwu, J. C., Huang, S. J., Guller, S., Karpisheva, K., Garabedian, M., et al. (2006). First-trimester trophoblast cell model gene response to hypoxia. Am. J. Obstet. Gynecol. 194, 687-693. doi: 10.1016/j.ajog.2006.01.067

Koppens, M. A., Bounova, G., Gargiulo, G., Tanger, E., Janssen, H., CornelissenSteijger, P., et al. (2016). Deletion of polycomb repressive complex 2 from mouse intestine causes loss of stem cells. Gastroenterology 151, 684-697. doi: 10.1053/j.gastro.2016.06.020

Korski, K. I., Kubli, D. A., Wang, B. J., Khalafalla, F. G., Monsanto, M. M., Firouzi, F., et al. (2019). Hypoxia prevents mitochondrial dysfunction and senescence in human c-kit(+) cardiac progenitor cells. Stem Cells 37, 555-567. doi: $10.1002 /$ stem. 2970

Kosan, C., Heidel, F. H., Godmann, M., and Bierhoff, H. (2018). Epigenetic erosion in adult stem cells: drivers and passengers of aging. Cells 7:237. doi: $10.3390 /$ cells 7120237

Kwon, S. Y., Chun, S. Y., Ha, Y. S., Kim, D. H., Kim, J., Song, P. H., et al. (2017). Hypoxia Enhances cell properties of human mesenchymal stem cells. Tissue Eng. Regen. Med. 14, 595-604. doi: 10.1007/s13770-017-0068-8

Larue, L., and Bellacosa, A. (2005). Epithelial-mesenchymal transition in development and cancer: role of phosphatidylinositol 3' kinase/AKT pathways. Oncogene 24, 7443-7454. doi: 10.1038/sj.onc.1209091

Laugesen, A., Hojfeldt, J. W., and Helin, K. (2016). Role of the polycomb repressive complex 2 (PRC2) in transcriptional regulation and cancer. Cold Spring Harb. Perspect. Med. 6:026575. doi: 10.1101/cshperspect.a026575

Lee, H. H., Chang, C. C., Shieh, M. J., Wang, J. P., Chen, Y. T., Young, T. H., et al. (2013). Hypoxia enhances chondrogenesis and prevents terminal differentiation through PI3K/Akt/FoxO dependent anti-apoptotic effect. Sci. Rep. 3:2683. doi: 10.1038/srep02683

Lee, P., Chandel, N. S., and Simon, M. C. (2020). Cellular adaptation to hypoxia through hypoxia inducible factors and beyond. Nat. Rev. Mol. Cell Biol. 21, 268-283. doi: 10.1038/s41580-020-0227-y

Lee, T. I., Jenner, R. G., Boyer, L. A., Guenther, M. G., Levine, S. S., Kumar, R. M., et al. (2006). Control of developmental regulators by Polycomb in human embryonic stem cells. Cell 125, 301-313. doi: 10.1016/j.cell.2006. 02.043

Leschik, J., Caron, L., Yang, H., Cowan, C., and Puceat, M. (2015). A view of bivalent epigenetic marks in two human embryonic stem cell lines reveals a different cardiogenic potential. Stem Cells Dev. 24, 384-392. doi: $10.1089 / \mathrm{scd} .2014 .0345$
Lewinska, A., Adamczyk-Grochala, J., Bloniarz, D., Horeczy, B., Zurek, S., Kurowicki, A., et al. (2020). Remifentanil preconditioning protects against hypoxia-induced senescence and necroptosis in human cardiac myocytes in vitro. Aging 12, 13924-13938. doi: 10.18632/aging.103604

Liu, L., Cheung, T. H., Charville, G. W., Hurgo, B. M., Leavitt, T., Shih, J., et al. (2013). Chromatin modifications as determinants of muscle stem cell quiescence and chronological aging. Cell Rep. 4, 189-204. doi: 10.1016/j.celrep.2013.05.043

Liu, W., Wen, Y., Bi, P., Lai, X., Liu, X. S., Liu, X., et al. (2012). Hypoxia promotes satellite cell self-renewal and enhances the efficiency of myoblast transplantation. Development 139, 2857-2865. doi: 10.1242/dev.079665

Liu, Y., Giannopoulou, E. G., Wen, D., Falciatori, I., Elemento, O., Allis, C. D., et al. (2016). Epigenetic profiles signify cell fate plasticity in unipotent spermatogonial stem and progenitor cells. Nat. Commun. 7:11275. doi: $10.1038 /$ ncomms11275

Maali, A., Atashi, A., Ghaffari, S., Kouchaki, R., Abdolmaleki, F., and Azad, M. (2018). A review on leukemia and iPSC technology: application in novel treatment and future. Curr. Stem Cell Res. Ther. 13, 665-675. doi: 10.2174/1574888X13666180731155038

Majmundar, A. J., Wong, W. J., and Simon, M. C. (2010). Hypoxiainducible factors and the response to hypoxic stress. Mol. Cell 40, 294-309. doi: 10.1016/j.molcel.2010.09.022

Martire, S., Gogate, A. A., Whitmill, A., Tafessu, A., Nguyen, J., Teng, Y. C., et al. (2019). Phosphorylation of histone $\mathrm{H} 3.3$ at serine 31 promotes $\mathrm{p} 300$ activity and enhancer acetylation. Nat. Genet. 51, 941-946. doi: 10.1038/s41588-019-0428-5

Mathieu, J., Zhang, Z., Nelson, A., Lamba, D. A., Reh, T. A., Ware, C., et al. (2013). Hypoxia induces re-entry of committed cells into pluripotency. Stem Cells 31, 1737-1748. doi: 10.1002/stem.1446

Mathieu, J., Zhou, W., Xing, Y., Sperber, H., Ferreccio, A., Agoston, Z., et al. (2014). Hypoxia-inducible factors have distinct and stage-specific roles during reprogramming of human cells to pluripotency. Cell Stem Cell 14, 592-605. doi: $10.1016 /$ j.stem.2014.02.012

Matoba, S., Wang, H., Jiang, L., Lu, F., Iwabuchi, K. A., Wu, X., et al. (2018). Loss of H3K27me3 imprinting in somatic cell nuclear transfer embryos disrupts post-implantation development. Cell Stem Cell 23, 343-354. doi: 10.1016/j.stem.2018.06.008

Matoba, S., and Zhang, Y. (2018). Somatic cell nuclear transfer reprogramming: mechanisms and applications. Cell Stem Cell 23, 471-485. doi: $10.1016 /$ j.stem.2018.06.018

Mattout, A., Biran, A., and Meshorer, E. (2011). Global epigenetic changes during somatic cell reprogramming to iPS cells. J. Mol. Cell Biol. 3, 341-350. doi: $10.1093 / \mathrm{jmcb} / \mathrm{mjr} 028$

Mimura, I., Nangaku, M., Kanki, Y., Tsutsumi, S., Inoue, T., Kohro, T., et al. (2012). Dynamic change of chromatin conformation in response to hypoxia enhances the expression of GLUT3 (SLC2A3) by cooperative interaction of hypoxia-inducible factor 1 and KDM3A. Mol. Cell. Biol. 32, 3018-3032. doi: 10.1128/MCB.06643-11

Moss, E. G., and Tang, L. (2003). Conservation of the heterochronic regulator Lin-28, its developmental expression and microRNA complementary sites. Dev. Biol. 258, 432-442. doi: 10.1016/S0012-1606(03)00126-X

Mosteiro, L., Pantoja, C., Alcazar, N., Marion, R. M., Chondronasiou, D., Rovira, M., et al. (2016). Tissue damage and senescence provide critical signals for cellular reprogramming in vivo. Science 354:aaf4445. doi: $10.1126 /$ science.aaf 4445

Mu, X., Xiang, G., Rathbone, C. R., Pan, H., Bellayr, I. H., Walters, T. J., et al. (2011). Slow-adhering stem cells derived from injured skeletal muscle have improved regenerative capacity. Am. J. Pathol. 179, 931-941. doi: 10.1016/j.ajpath.2011.05.004

Muz, B., de la Puente, P., Azab, F., and Azab, A. K. (2015). The role of hypoxia in cancer progression, angiogenesis, metastasis, and resistance to therapy. Hypoxia (Auckl) 3, 83-92. doi: 10.2147/HP.S93413

Nagoshi, N., Tsuji, O., Nakamura, M., and Okano, H. (2019). Cell therapy for spinal cord injury using induced pluripotent stem cells. Regen. Ther. 11, 75-80. doi: 10.1016/j.reth.2019.05.006

Nakade, K., Lin, C. S., Chen, X. Y., Tsai, M. H., Wuputra, K., Zhu, Z. W., et al. (2017). Jun dimerization protein 2 controls hypoxia-induced replicative senescence via both the p16(Ink4a)-pRb and Arf-p53 pathways. FEBS Open Bio 7, 1793-1804. doi: 10.1002/2211-5463.12325 
Pan, G., and Thomson, J. A. (2007). Nanog and transcriptional networks in embryonic stem cell pluripotency. Cell Res. 17, 42-49. doi: 10.1038/sj.cr.7310125

Park, J., Jun, E. K., Son, D., Hong, W., Jang, J., Yun, W., et al. (2019). Overexpression of Nanog in amniotic fluid-derived mesenchymal stem cells accelerates dermal papilla cell activity and promotes hair follicle regeneration. Exp. Mol. Med. 51:72. doi: 10.1038/s12276-0190266-7

Pasini, D., Bracken, A. P., Jensen, M. R., Lazzerini Denchi, E., and Helin, K. (2004). Suz12 is essential for mouse development and for EZH2 histone methyltransferase activity. EMBO J. 23, 4061-4071. doi: 10.1038/sj.emboj.7600402

Poljakova, J., Groh, T., Gudino, Z. O., Hrabeta, J., Borek-Dohalska, L., Kizek, R., et al. (2014). Hypoxia-mediated histone acetylation and expression of N-myc transcription factor dictate aggressiveness of neuroblastoma cells. Oncol. Rep. 31, 1928-1934. doi: 10.3892/or.2014.2999

Portela, A., and Esteller, M. (2010). Epigenetic modifications and human disease. Nat. Biotechnol. 28, 1057-1068. doi: 10.1038/nbt.1685

Prasad, P., Mittal, S. A., Chongtham, J., Mohanty, S., and Srivastava, T. (2017). Hypoxia-mediated epigenetic regulation of stemness in brain tumor cells. Stem Cells 35, 1468-1478. doi: 10.1002/stem.2621

Prickaerts, P., Adriaens, M. E., Beucken, T. V. D., Koch, E., Dubois, L., Dahlmans, V. E. H., et al. (2016). Hypoxia increases genome-wide bivalent epigenetic marking by specific gain of H3K27me3. Epigenet. Chromatin 9:46. doi: 10.1186/s13072-016-0086-0

Rankin, E. B., Giaccia, A. J., and Schipani, E. (2011). A central role for hypoxic signaling in cartilage, bone, and hematopoiesis. Curr. Osteoporos. Rep. 9, 46-52. doi: 10.1007/s11914-011-0047-2

Rybouchkin, A., Kato, Y., and Tsunoda, Y. (2006). Role of histone acetylation in reprogramming of somatic nuclei following nuclear transfer. Biol. Reprod. 74, 1083-1089. doi: 10.1095/biolreprod.105.047456

Sadaghianloo, N., Contenti, J., Dufies, M., Parola, J., Rouleau, M., Lee, S., et al. (2020). Co-culture of human fibroblasts, smooth muscle and endothelial cells promotes osteopontin induction in hypoxia. J. Cell. Mol. Med. 24, 2931-2941. doi: $10.1111 /$ jcmm. 14905

Schrode, N., Xenopoulos, P., Piliszek, A., Frankenberg, S., Plusa, B., and Hadjantonakis, A. K. (2013). Anatomy of a blastocyst: cell behaviors driving cell fate choice and morphogenesis in the early mouse embryo. Genesis 51, 219-233. doi: $10.1002 /$ dvg. 22368

Scully, D., Keane, E., Batt, E., Karunakaran, P., Higgins, D. F., and Itasaki, N. (2016). Hypoxia promotes production of neural crest cells in the embryonic head. Development 143, 1742-1752. doi: 10.1242/dev.131912

Shan, Y., Liang, Z., Xing, Q., Zhang, T., Wang, B., Tian, S., et al. (2017). PRC2 specifies ectoderm lineages and maintains pluripotency in primed but not naive ESCs. Nat. Commun. 8:672. doi: 10.1038/s41467-017-00668-4

Shipony, Z., Mukamel, Z., Cohen, N. M., Landan, G., Chomsky, E., Zeliger, S. R., et al. (2014). Dynamic and static maintenance of epigenetic memory in pluripotent and somatic cells. Nature 513, 115-119. doi: 10.1038/nature13458

Shyh-Chang, N., Zhu, H., Yvanka de Soysa, T., Shinoda, G., Seligson, M. T., Tsanov, K. M., et al. (2013). Lin28 enhances tissue repair by reprogramming cellular metabolism. Cell 155, 778-792. doi: 10.1016/j.cell.2013.09.059

Song, H. J., Chen, X., Jiao, Q., Qiu, Z. L., Shen, C. T., Zhang, G. Q., et al. (2020). HIF-lalpha-mediated TERT activation inducing autophagy through mTOR promotes papillary thyroid carcinoma progression during hypoxia stress. Thyroid. doi: 10.1089/thy.2020.0023. [Epub ahead of print].

Stojic, L., Jasencakova, Z., Prezioso, C., Stutzer, A., Bodega, B., Pasini, D., et al. (2011). Chromatin regulated interchange between polycomb repressive complex 2 (PRC2)-Ezh2 and PRC2-Ezh1 complexes controls myogenin activation in skeletal muscle cells. Epigenet. Chromatin 4:16. doi: $10.1186 / 1756-8935-4-16$

Takahashi, K., Okita, K., Nakagawa, M., and Yamanaka, S. (2007). Induction of pluripotent stem cells from fibroblast cultures. Nat. Protoc. 2, 3081-3089. doi: 10.1038/nprot.2007.418

Takahashi, K., and Yamanaka, S. (2006). Induction of pluripotent stem cells from mouse embryonic and adult fibroblast cultures by defined factors. Cell 126, 663-676. doi: 10.1016/j.cell.2006.07.024

Takasawa, K., Arai, Y., Yamazaki-Inoue, M., Toyoda, M., Akutsu, H., Umezawa, A., et al. (2018). DNA hypermethylation enhanced telomerase reverse transcriptase expression in human-induced pluripotent stem cells. Hum. Cell 31, 78-86. doi: 10.1007/s13577-017-0190-x

Tatebayashi, K., Tanaka, Y., Nakano-Doi, A., Sakuma, R., Kamachi, S., Shirakawa, M., et al. (2017). Identification of multipotent stem cells in human brain tissue following stroke. Stem Cells Dev. 26, 787-797. doi: 10.1089/scd.20 16.0334

Teichroeb, J. H., Kim, J., and Betts, D. H. (2016). The role of telomeres and telomerase reverse transcriptase isoforms in pluripotency induction and maintenance. RNA Biol. 13, 707-719. doi: 10.1080/15476286.2015.11 34413

Thomson, J. A., Itskovitz-Eldor, J., Shapiro, S. S., Waknitz, M. A., Swiergiel, J. J., Marshall, V. S., et al. (1998). Embryonic stem cell lines derived from human blastocysts. Science 282, 1145-1147. doi: 10.1126/science.282.5391.1145

Tsai, C. C., Chen, Y. J., Yew, T. L., Chen, L. L., Wang, J. Y., Chiu, C. H., et al. (2011). Hypoxia inhibits senescence and maintains mesenchymal stem cell properties through down-regulation of E2A-p21 by HIF-TWIST. Blood 117, 459-469. doi: 10.1182/blood-2010-05-287508

Tsuji, K., Kitamura, S., and Makino, H. (2014). Hypoxia-inducible factor lalpha regulates branching morphogenesis during kidney development. Biochem. Biophys. Res. Commun. 447, 108-114. doi: 10.1016/j.bbrc.2014.03.111

Veneti, Z., Gkouskou, K. K., and Eliopoulos, A. G. (2017). Polycomb repressor complex 2 in genomic instability and cancer. Int. J. Mol. Sci. 18:1657. doi: 10.3390/ijms 18081657

Vojnits, K., Pan, H., Dai, X., Sun, H., Tong, Q., Darabi, R., et al. (2017). Functional neuronal differentiation of injury-induced musclederived stem cell-like cells with therapeutic implications. Sci. Rep. 7:1177. doi: 10.1038/s41598-017-01311-4

Vojnits, K., Pan, H., Mu, X., and Li, Y. (2015). Characterization of an injury induced population of muscle-derived stem cell-like cells. Sci. Rep. 5:17355. doi: 10.1038/srep17355

Wang, C., Liu, X., Gao, Y., Yang, L., Li, C., Liu, W., et al. (2018). Reprogramming of H3K9me3-dependent heterochromatin during mammalian embryo development. Nat. Cell Biol. 20, 620-631. doi: 10.1038/s41556-018-0093-4

Wang, P., Wan, W., Xiong, S., Wang, J., Zou, D., Lan, C., et al. (2017a). HIF $1 \alpha$ regulates glioma chemosensitivity through the transformation between differentiation and dedifferentiation in various oxygen levels. Sci. Rep. 7:7965. doi: 10.1038/s41598-017-06086-2

Wang, P., Wan, W. W., Xiong, S. L., Feng, H., and Wu, N. (2017b). Cancer stem-like cells can be induced through dedifferentiation under hypoxic conditions in glioma, hepatoma and lung cancer. Cell Death Discov. 3:16105. doi: $10.1038 /$ cddiscovery.2016.105

Wang, S., Sun, Y., Ren, R., Xie, J., Tian, X., Zhao, S., et al. (2019). H3K27me3 depletion during differentiation promotes myogenic transcription in porcine satellite cells. Genes 10:231. doi: 10.3390/genes10030231

Wang, Y., Shi, S., Liu, H., and Meng, L. (2016). Hypoxia enhances direct reprogramming of mouse fibroblasts to cardiomyocyte-like cells. Cell. Reprogram. 18, 1-7. doi: 10.1089/cell.2015.0051

Wilmut, I., Beaujean, N., de Sousa, P. A., Dinnyes, A., King, T. J., Paterson, L. A., et al. (2002). Somatic cell nuclear transfer. Nature 419, 583-586. doi: 10.1038/nature01079

Wilmut, I., Schnieke, A. E., McWhir, J., Kind, A. J., and Campbell, K. H. (1997). Viable offspring derived from fetal and adult mammalian cells. Nature 385 , 810-813. doi: 10.1038/385810a0

Wu, M. Z., Tsai, Y. P., Yang, M. H., Huang, C. H., Chang, S. Y., Chang, C. C., et al. (2011). Interplay between HDAC3 and WDR5 is essential for hypoxia-induced epithelial-mesenchymal transition. Mol. Cell 43, 811-822. doi: 10.1016/j.molcel.2011.07.012

Xing, J., Ying, Y., Mao, C., Liu, Y., Wang, T., Zhao, Q., et al. (2018). Hypoxia induces senescence of bone marrow mesenchymal stem cells via altered gut microbiota. Nat. Commun. 9:2020. doi: 10.1038/s41467-018-04453-9

Yang, G., Zhang, L., Liu, W., Qiao, Z., Shen, S., Zhu, Q., et al. (2020). Dux-mediated corrections of aberrant $\mathrm{H} 3 \mathrm{~K} 9 \mathrm{ac}$ during 2-cell genome activation optimize efficiency of somatic cell nuclear transfer. Cell Stem Cell 28, 150-163.e5. doi: $10.1016 /$ j.stem.2020.09.006

Yang, X., Yang, S., Wang, C., and Kuang, S. (2017). The hypoxia-inducible factors HIF1alpha and HIF2alpha are dispensable for embryonic muscle development but essential for postnatal muscle regeneration. J. Biol. Chem. 292, 5981-5991. doi: $10.1074 /$ jbc.M116.756312 
Yoshida, Y., Takahashi, K., Okita, K., Ichisaka, T., and Yamanaka, S. (2009). Hypoxia enhances the generation of induced pluripotent stem cells. Cell Stem Cell 5, 237-241. doi: 10.1016/j.stem.2009.08.001

Yu, J., Vodyanik, M. A., Smuga-Otto, K., Antosiewicz-Bourget, J., Frane, J. L., Tian, S., et al. (2007). Induced pluripotent stem cell lines derived from human somatic cells. Science 318, 1917-1920. doi: 10.1126/science.1151526

Yu, R. M., Chen, E. X., Kong, R. Y., Ng, P. K., Mok, H. O., and Au, D. W. (2006). Hypoxia induces telomerase reverse transcriptase (TERT) gene expression in non-tumor fish tissues in vivo: the marine medaka (Oryzias melastigma) model. BMC Mol. Biol. 7:27. doi: 10.1186/1471-2199-7-27

Yu, Y., Deng, P., Yu, B., Szymanski, J. M., Aghaloo, T., Hong, C., et al. (2018). Inhibition of EZH2 promotes human embryonic stem cell differentiation into mesoderm by reducing H3K27me3. Stem Cell Rep. 11, 1579-1580. doi: 10.1016/j.stemcr.2018.11.013

Yui, S., Azzolin, L., Maimets, M., Pedersen, M. T., Fordham, R. P., Hansen, S. L., et al. (2018). YAP/TAZ-dependent reprogramming of colonic epithelium links ECM remodeling to tissue regeneration. Cell Stem Cell 22, 35-49. doi: 10.1016/j.stem.2017.11.001

Zhang, Z., Zhang, L., Zhou, Y., Li, L., Zhao, J., Qin, W., et al. (2019). Increase in HDAC9 suppresses myoblast differentiation via epigenetic regulation of autophagy in hypoxia. Cell Death Dis. 10:552. doi: 10.1038/s41419-0191763-2

Zhu, J., Wang, K., Li, T., Chen, J., Xie, D., Chang, X., et al. (2017). Hypoxia-induced TET1 facilitates trophoblast cell migration and invasion through HIF1alpha signaling pathway. Sci. Rep. 7:8077. doi: 10.1038/s41598-017-07560-7

Conflict of Interest: YL was one of the executive editors for Journal of Cellular BioChemistry.

The remaining authors declare that the research was conducted in the absence of any commercial or financial relationships that could be construed as a potential conflict of interest.

Copyright (c) 2021 Nakamura, Shi, Darabi and Li. This is an open-access article distributed under the terms of the Creative Commons Attribution License (CC BY). The use, distribution or reproduction in other forums is permitted, provided the original author(s) and the copyright owner(s) are credited and that the original publication in this journal is cited, in accordance with accepted academic practice. No use, distribution or reproduction is permitted which does not comply with these terms. 